\title{
Coronary microemboli have long-term effects on regional left ventricular function: MRI 3D strain analysis Robert Jablonowski*1, Marcus Carlsson ${ }^{1,2}$, Alastair J Martin ${ }^{2}$ and Maythem Saeed ${ }^{2}$
}

Address: ${ }^{1}$ Dep of Clinical Physiology, Lund, Sweden and ${ }^{2}$ Dep of Radiology and Biomedical Imaging, San Francisco, CA, USA

* Corresponding author

from 13th Annual SCMR Scientific Sessions

Phoenix, AZ, USA. 21-24 January 2010

Published: 21 January 2010

Journal of Cardiovascular Magnetic Resonance 20 I0, I2(SuppI I):P57 doi: I0.I I86/I532-429X-I2-SI-P57

This abstract is available from: http://jcmr-online.com/content/I2/SI/P57

(C) 2010 Jablonowski et al; licensee BioMed Central Ltd.

\section{Introduction}

Microembolization is common during coronary intervention in patients. The long-term effects of microemboli on regional 3D strain and global left ventricular (LV) function have not been examined.

\section{Purpose}

This experimental study investigated whether coronary microemboli have long-term deleterious effects on LV function using MRI strain analysis.

\section{Methods}

A hybrid X-ray and MRI system was used to catheterize the LAD in 6 pigs and deliver the embolic materials (40-120 $\mu \mathrm{m}, 250,000$ count). The area at risk (AAR) was determined on first-pass perfusion (FPP) MRI before and after microembolization. Delayed enhancement (DE)-MRI and histochemical staining were used to visualize and measure microinfarcts. Analysis of longitudinal strain was performed on phase-contrast MRI and radial strain as well as global function on ssfp MRI using Segment http://seg ment.heiberg.se. Tagged MRI were analyzed to measure circumferential strain using HARP. The Student's t-test was used to determine if strain over the cardiac cycle differed between baseline, acute ( $1 \mathrm{~h}$ ) and chronic phases (78 weeks).

\section{Results}

Coronary microemboli caused an acute reduction in ejection fraction $(32 \pm 3 \%$ vs. $50 \pm 3 \%, P<0.05)$ compared to baseline. Longitudinal strain in the AAR and remote acutely declined compared to baseline $(P<0.001$, Figure), suggesting that microemboli has acute global effects. At the chronic phase the AAR displayed a partial and remote a full recovery of function compared to baseline (Figure). Radial strain also declined in the AAR ( $1.0 \pm 7.2 \%$ vs. 58.1 $\pm 6.3 \%, \mathrm{P}<0.004)$ and remote $(17.8 \pm 6.8 \%$ vs. $51.1 \pm$ $5.8 \%, \mathrm{P}<0.01)$ at the acute phase compared to baseline. There was a persistent decrease in the AAR but not in remote at the chronic phase $(P<0.001$ and $P=0.18)$. On the contrary, there was a persistent decline in circumferential strain at the acute and chronic phase in the AAR compared to baseline, as well as in remote area $(\mathrm{P}<0.001$, Figure 1). FPP, DE-MRI and histopathology at postmortem confirmed the presence of microembolization and microinfarction. Microinfarcts could be detected in the acute phase as a perfusion defect on FPP but not on DEMRI $(0.1 \pm 0.0 \% L V)$. No perfusion defect could be visually detected on FPP at the chronic phase but DE-MRI showed speckled hyperenhancement in the AAR. The microinfarct size was $6.6 \pm 0.5 \% \mathrm{LV}$ mass.

\section{Conclusion}

This serial MRI study demonstrated a persistent regional LV dysfunction in the microembolized area. Regional 3D strain measurements may be useful in predicting decreased contractility in the LV in patients with biomarker release after coronary intervention.

${ }^{*} P<0.05,{ }^{* * *} P<0.001$ compared to baseline and $\dagger \mathrm{p}<$ 0.05 compared with AAR at the same stage. 

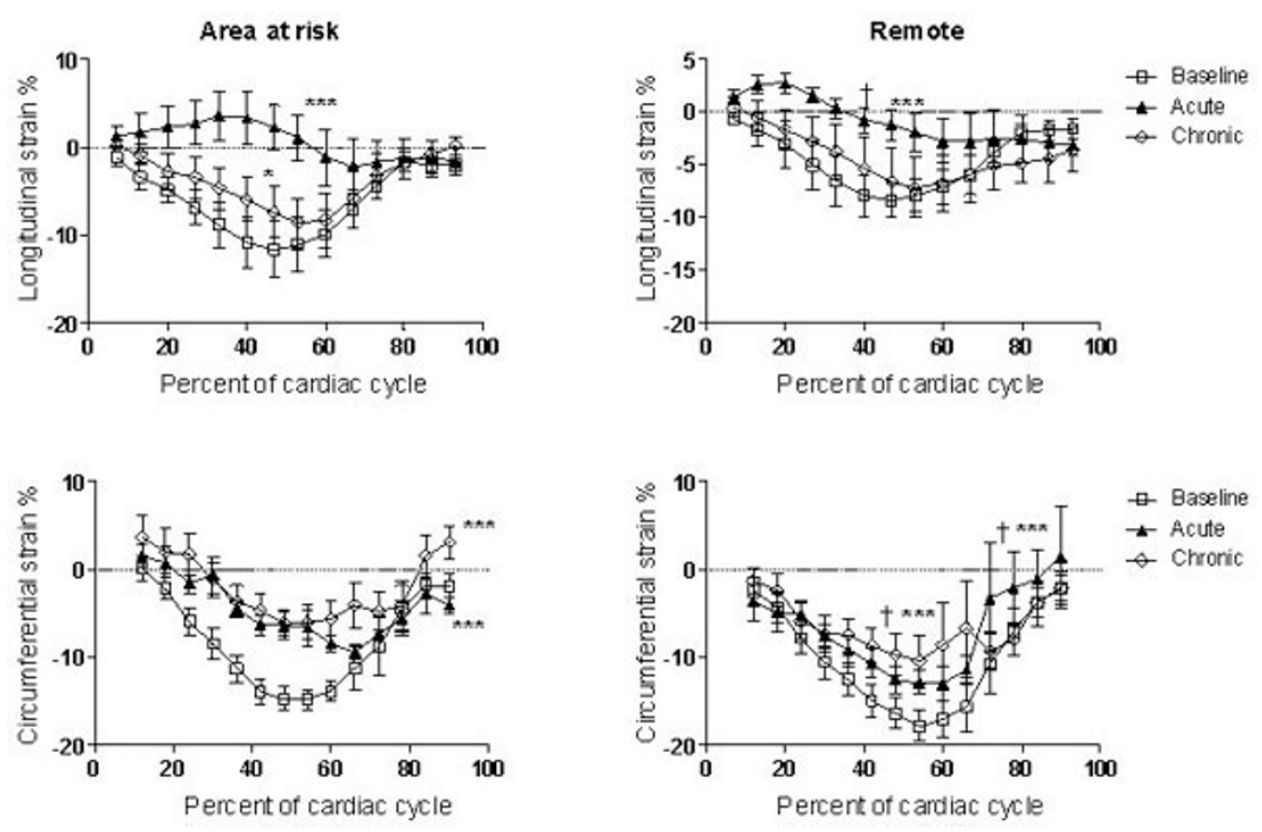

Figure I 